\title{
Phase retrieval approach for an accurate reconstruction of an arbitrary optical waveform in the petahertz optical oscilloscope
}

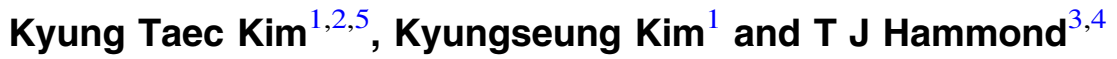 \\ ${ }^{1}$ Center for Relativistic Laser Science, Institute for Basic Science, 123 Cheomdangwagiro, Buk-gu, \\ Gwangju, 61005, Korea \\ ${ }^{2}$ Department of Physics and Photon Science, Gwangju Institute of Science and Technology, 123 \\ Cheomdangwagiro, Buk-gu, Gwangju, 61005, Korea \\ ${ }^{3}$ Joint Attosecond Science Laboratory, National Research Council and University of Ottawa, Ottawa, ON, \\ K1A 0R6, Canada \\ ${ }^{4}$ Institute for the Frontier of Attosecond Science and Technology, CREOL and Department of Physics, \\ University of Central Florida, Orlando, FL 32816, USA
}

E-mail: kyungtaec@gist.ac.kr

Received 10 August 2016, revised 8 November 2016

Accepted for publication 15 November 2016

Published 3 January 2017

\begin{abstract}
An arbitrary optical waveform can be measured using the technique called the 'petahertz optical oscilloscope' in which an electron trajectory in the process of high harmonic generation is used as a fast temporal gate. In the petahertz optical oscilloscope, a signal laser pulse that needs to be measured is superposed on a fundamental laser pulse that generates high harmonic radiation. The addition of the signal laser pulse slightly modifies the amplitude and phase of the harmonic radiation, which have information on the signal field. While these changes are imposed in the generation medium, the harmonic radiation is measured at the detector. Thus, one needs a special technique to find the phase modulation in the medium using the variation of the spatiallyresolved harmonic spectrum measured at the far field. We find that a simple estimation using the deflection angle of the harmonic radiation is not accurate enough for a broadband signal pulse. Here we introduce a phase retrieval approach for the reconstruction of the broadband signal pulse. Our calculations show that the broadband signal pulse can be accurately reconstructed using the phase retrieval approach.
\end{abstract}

Keywords: laser pulse characterization, high harmonic generation, attosecond pulse generation (Some figures may appear in colour only in the online journal)

\section{Introduction}

The temporal characterization of laser pulses is an important subject for studying light-matter interactions. An arbitrary

\footnotetext{
5 This article belongs to the special issue: emerging leaders, which features invited work from the best early-career researchers working within the scope of J. Phys. B. This project is part of the Journal of Physics series' 50th anniversary celebrations in 2017. Kyung Taec Kim was selected by the Editorial Board of $J$. Phys. B as an Emerging Leader.
}

time-dependent field of ultrashort laser pulses can now be measured by two approaches: the attosecond streak camera $[1,2]$, and the petahertz optical oscilloscope [3]. Both techniques use the process of high harmonic generation [4]. The attosecond streak camera uses an isolated attosecond pulse obtained from high harmonic radiation to probe the timedependent field. The petahertz optical oscilloscope uses the electron trajectories in the process of high harmonic generation for the measurement. 
In the attosecond streak camera, an attosecond pulse photo-ionizes an electron in the presence of the weak laser field which we call a signal field. Since the electron experiences the signal field from the moment of ionization, it may gain or lose momentum depending on the relative time delay between the attosecond pulse and the signal field. Thus, one can completely determine the signal field by measuring the momentum shift of the photoelectron spectrum as a function of the time delay between the attosecond pulse and the laser pulse $[2,5]$.

One can roughly characterize the signal field from the momentum shift of the photoelectron spectra [2]. However, there is a more elegant way to extract information on the signal field from the photoelectron spectra, which is known as the frequency-resolved optical gating for complete reconstruction of attosecond bursts (FROG CRAB) [6]. The FROG CRAB is a phase retrieval approach that is initially developed for the characterization of attosecond pulses. However, the signal field can also be retrieved very accurately. Since the reconstruction algorithm uses a full two-dimensional (energy and time delay) data which is called a FROG trace, the reconstruction results obtained using the phase retrieval approach are very accurate and robust [7, 8].

In the petahertz optical oscilloscope, we use the electron trajectory in the process of high harmonic generation as a fast temporal gate. Two laser pulses are used in the technique: the signal and the fundamental pulses. The weak signal field-the field that is to be measured-acts as a perturbation on the fundamental laser field that generates the high harmonic radiation. This signal field slightly alters the amplitude and phase of the high harmonic radiation. The phase shift of the harmonic radiation is proportional to the signal field. Therefore, the signal field can be determined if the phase shift of the harmonic radiation in the medium can be found using the variation of the spatial distribution measured at the far field [9]. A simple approach to find the phase shift of the harmonic radiation using the shift of the spatial distribution fails when the signal pulse has a broadband spectrum. In this work, we introduce the phase retrieval approach to retrieve information on the signal field from the spatial distribution of the harmonic radiation measured in the petahertz optical oscilloscope. We show that the broadband signal pulse can be accurately reconstructed using the phase retrieval approach.

\section{Petahertz optical oscilloscope}

\subsection{Phase retrieval approach}

High harmonic generation can be described by the three step model [4]. First, an electron can be liberated through tunneling ionization when intense fundamental laser field is irradiated on an atom. Second, the electron is accelerated after the ionization, and it is driven back to the parent ion in the next half cycle when the sign of the fundamental field is changed. When the electron recombines with the parent ion, the harmonic radiation is emitted with an energy of the

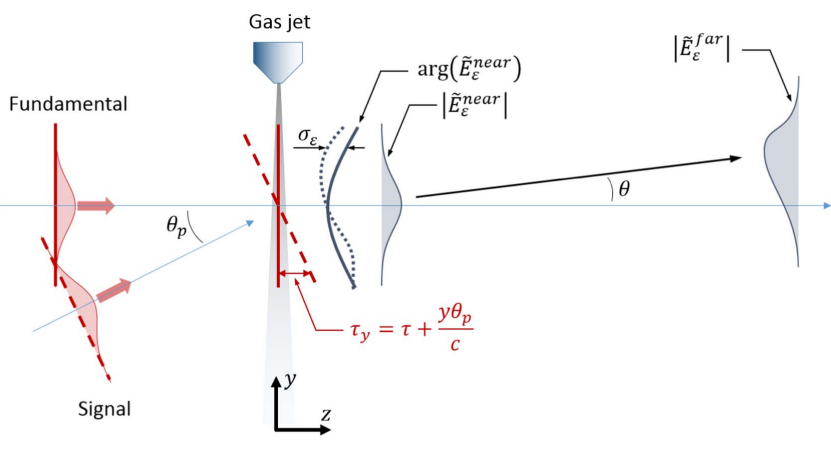

Figure 1. Non-collinear setup for the petahertz optical oscilloscope measurement. The signal laser pulse is superposed on the fundamental laser pulse in a small angle $\theta_{p}$ in the medium. The delay between two laser pulses is position-dependent. The original phase of the harmonic radiation, $\arg \left(\tilde{E}_{\varepsilon}^{\text {near }}\right)$, is modified by $\sigma_{\varepsilon}$ which in turn changes the propagation angle of the harmonic radiation. The spatial distribution of the far-field harmonic radiation $\left|\tilde{E}_{\varepsilon}^{\text {far }}(\theta)\right|$ is measured as a function of the propagation angle $\theta$ and time delay $\tau$.

electron kinetic energy plus the ionization potential of the atom.

The petahertz optical oscilloscope uses the electron trajectory in the process of high harmonic generation to measure the signal field. When a weak signal field is added in the fundamental field, it slightly alters the ionization time, the electron trajectory, and the recombination time. Thus, the amplitude and phase of the harmonic radiation are changed. While the amplitude of the harmonic radiation is changed mainly due to the change of the ionization probability at the ionization moment, the phase shift of the harmonic radiation is caused by the change of the action accumulated during the excursion of the electron (from the ionization to the recombination) and by the change of the recombination time [9-11].

The spatial distribution of the harmonic beam at the far field (at the detector) is more sensitive to the phase change than the amplitude change in the near field (in the medium). This phase shift is imposed by the superposition of the signal field. Thus, it changes as a function of the time delay between the fundamental and the signal pulse, which is position dependent as $\left(\tau+y \theta_{p} / c\right)$ in the non-collinear geometry shown in figure 1. Here, $\tau$ is the delay of the signal pulse at $y=0$. The phase modulation $\sigma_{\varepsilon}$ is also delayed depending on the observation energy $\varepsilon$, which is related with the emission time of the harmonic radiation. If one compares the delay of the phase modulation over a broad energy range, one can access information on the intrinsic chirp (also called attochirp) of the attosecond pulse [9]. However, this energy dependent delay can be neglected in this work because the whole pulse of the reconstructed signal is delayed. It only gives a constant time delay, which does not affect the temporal shape of the reconstructed signal field. Thus, the phase shift can be written in the length scale as $\sigma_{\varepsilon}\left(y-y_{d}\right)$ using $y_{d}=-c \tau / \theta_{p}$.

The spatial distribution of the harmonic beam at the far field can be expressed as the Fourier transform of the near 

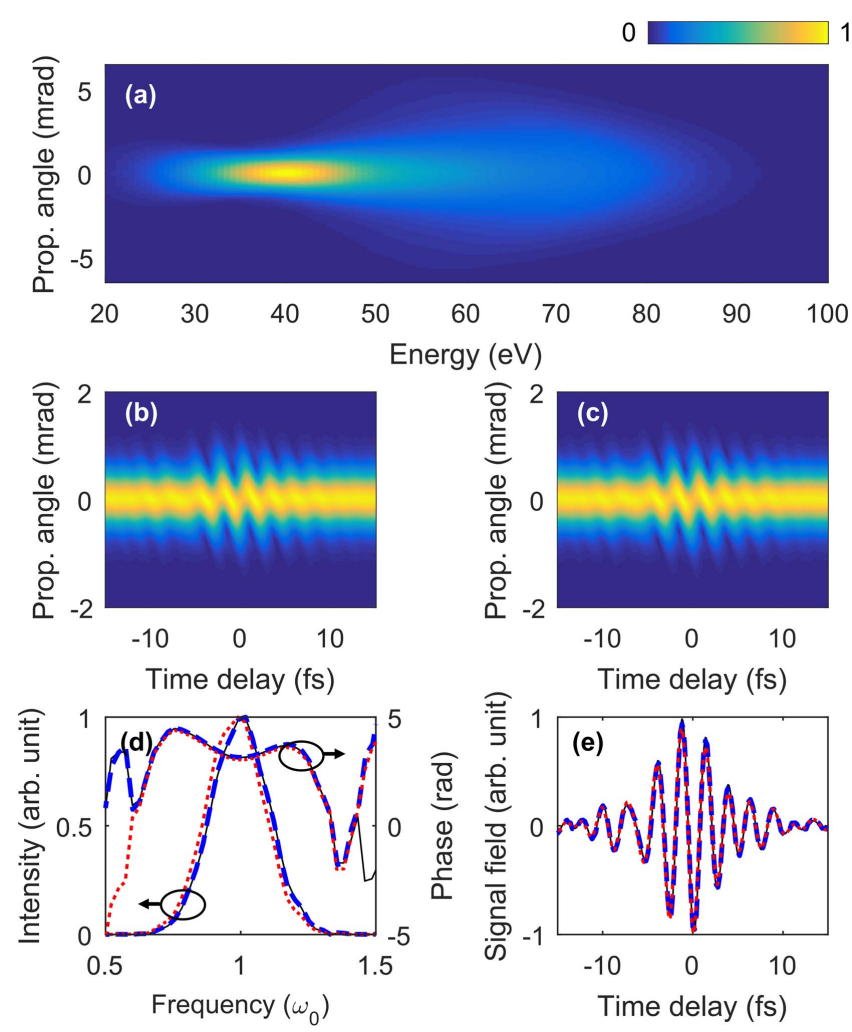

Figure 2. Reconstruction of a 5.4 fs signal pulse. (a) Spatiallyresolved harmonic spectrum calculated from $\mathrm{Ne}$ atoms in the far field using the strong field approximation model. The central wavelength of the fundamental and the signal pulse are $800 \mathrm{~nm}$. The intensity of the fundamental is $3.0 \times 10^{14} \mathrm{~W} \mathrm{~cm}^{-2}$. (b) Spatial distribution of harmonic beam at $40 \mathrm{eV}$ as a function of time delay. (c) The reconstructed signal fields using the PCGP algorithm after 500 iterations. (d) The spectra and spectral phases of the original (black solid line) and reconstructed signal pulses using Gaussian fitting (red dotted line) and PCGP algorithm (blue dashed line). (e) The electric field of the original (black solid line) and the reconstructed signal pulses using Gaussian fitting (red dotted line) and PCGP algorithm (blue dashed line).

\section{field harmonic beam as}

$$
\tilde{E}_{\varepsilon}^{\mathrm{far}}\left(\theta, y_{\mathrm{d}}\right)=\int_{-\infty}^{+\infty} \tilde{E}_{\varepsilon}^{\text {near }}(y) G_{\varepsilon}\left(y-y_{d}\right) \exp \left(\mathrm{i} k_{\varepsilon} \theta y\right) \mathrm{d} y
$$

Here, $\theta$ is the propagation angle, $k_{\varepsilon}$ is the wave number of the harmonic radiation at the observation energy $\varepsilon . \tilde{E}_{\varepsilon}^{\text {near }}$ and $\tilde{E}_{\varepsilon}^{\text {far }}$ are the spectral amplitudes of the harmonic beam in the near and far field, respectively. Note that the phase retrieval problem is solved in space domain in which the angular spatial frequency is defined as $k_{\varepsilon} \theta$. However, the FROG trace is expressed with the propagation angle $\theta$ to represent with a physical quantity. The function $G_{\varepsilon}\left(y-y_{d}\right)$ describes both the amplitude and the phase modulation imposed by the superposition of the signal field. The phase of the $G_{\varepsilon}\left(y-y_{d}\right)$ is the phase shift of the harmonic radiation [i.e. $\left.\sigma_{\varepsilon}=\arg G_{\varepsilon}(y)\right]$.

As we change the time delay, the far-field spatial distribution of the harmonic beam is changed due to the phase gradient imposed on the near-field harmonic beam. One can directly estimate the first-order phase modulation $(\mathrm{d} \sigma / \mathrm{d} y)$ from the shift of the far-field harmonic beam [3]. However, there are two potential problems in this simple approach.
First, the wavefront of the harmonic beam can be curved $[9,12]$. When the sinusoidal phase modulation is imposed on the curved wavefront, the spatial distribution of the harmonic radiation is shifted due to the first-order phase modulation. At the same time, however, the divergence of the harmonic beam can also be changed due to the second-order phase modulation of the oscillating signal field $\left(\mathrm{d}^{2} \sigma / \mathrm{d} y^{2}\right)$. Thus, the simple estimation based on the first-order approximation would produce artifacts in the reconstruction. Second, the harmonic beam can be diffracted if multi-cycle phase modulation is imposed on the harmonic beam. If the harmonic beam is diffracted, the phase information will be lost. The multi cycle phase modulation can be imposed if the signal wavelength is short or the superposition angle $\theta_{p}$ is too large. For a broadband signal, a simple waveform estimation based on the firstorder approximation would result in systematic errors in the waveform measurement. Thus, a reliable method to reconstruct the signal field from the spatial distribution of the harmonic beam is required.

It should be noted that the equation (1) has the form of the spectrogram in which the amplitude and phase modulation imposed by the signal field works as a temporal gate function $G_{\varepsilon}(y)$. The spatial distribution at the far field measured as a function of time delay, $\tilde{E}_{\varepsilon}^{\text {far }}\left(\theta, y_{d}\right)$, is the FROG trace to solve. Since one can only measure the absolute value of the spatial distributions, $\left|\tilde{E}_{\varepsilon}^{f a r}\left(\theta, y_{d}\right)\right|$, in the experiment, a phase retrieval algorithm is required. A blind FROG algorithm such as the principal component generalized projection (PCGP) algorithm can be applied to find both the near-field harmonic radiation $\tilde{E}_{\varepsilon}^{\text {near }}\left(\theta, y_{d}\right)$ and the gate function $G_{\varepsilon}(y)[7,8]$.

The signal field can be obtained from the phase shift $\sigma_{\varepsilon}$. For the long wavelength signal, the wavelength of the signal field is much longer than the duration of the electron trajectory. The phase shift is simply proportional to the signal field [i.e. $\sigma_{\varepsilon} \propto E_{s}(t)$ ]. However, if the signal field contains short wavelength components, the single atom response to the signal field should be taken into account [13]. The proportional constant is a complex number that depends on the signal wavelength. The signal field can be obtained from the relation

$$
\sigma_{\varepsilon}=\int A_{\omega} \exp \left(\mathrm{i} \Phi_{\omega}\right) \tilde{E}_{s}(\omega) \exp (\mathrm{i} \omega t) \mathrm{d} \omega
$$

Here, $A_{\omega}$ and $\Phi_{\omega}$ are the amplitude and phase correction factors that can be obtained from the theoretical calculations [13].

\subsection{SFA model description}

To demonstrate the reconstruction of the signal field, we calculate the far-field spectrum of the harmonic radiation. The near-field harmonic radiation is calculated using the strong field approximation model in which the ionization rate is obtained by the Yudin-Ivanov model [14], and the electron trajectories are classically solved [15]. High harmonic radiation generated from a half optical cycle is selected to mimic the generation of the isolated attosecond pulses from atoms distributed along a line in the transverse direction $y$ as shown in figure 1 . The spectral amplitude of the near-field harmonic 
radiation, $\tilde{E}_{\varepsilon}^{\text {near }}(y)$, is obtained by taking the Fourier transform of the harmonic radiation $\tilde{E}_{t}^{\text {near }}(y)$ in time domain. Then, the harmonic spectrum $\tilde{E}_{\varepsilon}^{\text {far }}(\theta)$ is obtained by taking the Fourier transform of $\tilde{E}_{\varepsilon}^{\text {near }}(y)$ along the transverse direction for all XUV photon energies. Figure 2(a) shows the harmonic spectrum calculated from $\mathrm{Ne}$ atoms. The signal pulse has a complicated temporal shape. The duration of the signal pulse is $5.4 \mathrm{fs}$ in the full width at half maximum. The center wavelength is $800 \mathrm{~nm}$ for both the fundamental and the signal field. The beam size of the fundamental is $30 \mu \mathrm{m}$.

To see a clear modulation of the propagation angle of the harmonic beam, the angle between two pulses $\theta_{p}$ should be optimized according to the signal wavelength $\lambda_{\mathrm{s}}$. If $\theta_{p}$ is too small, the deflection of the harmonic beam will be small. If $\theta_{p}$ is too big, the harmonic beam will be diffracted and the phase information will vanish. We find that a clear shift of the harmonic beam is observed when the phase gradient of a half wavelength of the signal field is imposed on harmonic beam. Thus, the angle of the signal beam is selected by the relation

$$
\theta_{p} \sim \frac{\lambda_{s}}{2 w_{X}}
$$

Here, $w_{X}$ is the beam size of the harmonic beam. In the case shown in figure 2, the harmonic beam size is $14.6 \mathrm{um}$ at $40 \mathrm{eV}$ and the signal wavelength is centered at $800 \mathrm{~nm}$. Thus, the angle of the signal pulse is set to be $\theta_{p}=20 \mathrm{mrad}$.

After the superposition of the signal field, harmonic spectra are calculated as a function of time delay. Vertical lineouts of the harmonic spectra at the observation energy of $40 \mathrm{eV}$ are added side by side as a function of time delay as shown in figure 2(b). The signal pulse can be reconstructed by two different ways. First, we reconstruct the signal pulse by finding out the shift of the spatial distribution of harmonics at the far field. The center of the Gaussian distribution was fitted for each time delay to obtain $\mathrm{d} \sigma_{\varepsilon} / \mathrm{d} \tau$ and then it was integrated to get $\sigma_{\varepsilon}$ as shown in figures 2(d)-(e). Second, $\sigma_{\varepsilon}$ obtained by applying PCGP algorithm is also shown in figures 2(d)-(e). The reconstructed FROG trace in figure 2(c) is almost identical with the trace obtained by the SFA calculations in figure 2(b). In both cases, the single atom response is taken into account using the equation (2) to find the signal pulse from the phase shift $\sigma_{\varepsilon}$. For this signal pulse, both approaches accurately reconstruct the amplitude and phase of the signal pulse as shown in figures 2(d) and (e).

Now we examine the case of the signal pulse that has a broad bandwidth. The FROG trace is calculated with a positively-chirped $2.3 \mathrm{fs}$ signal pulse as shown in figure 3(a). The reconstructed FROG trace obtained using the PCGP algorithm is in figure 3(b) which shows an identical shape to figure 3(a). The reconstructed spectral amplitude and phases are shown in figure 3(c). Unlike the relatively narrow bandwidth signal shown in figure 2 , the signal field obtained using Gaussian fitting fails to reconstruct the original signal field. However, the reconstruction obtained using PCGP algorithm reproduces the overall shape of the spectral intensity and phases. The reconstructed temporal profile of the signal fields are shown in figure 3(d). The electric field of the signal field is very well reconstructed using PCGP algorithm whereas the
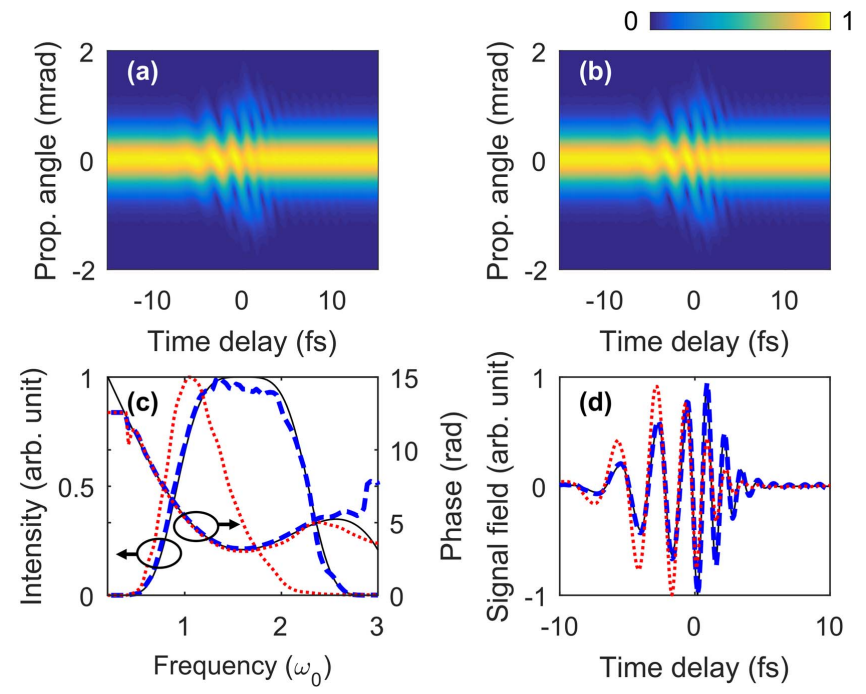

Figure 3. Reconstruction of a 2.3 fs signal pulse. (a) The FROG trace calculated using the SFA model from Ne atoms at the intensity of $3.0 \times 10^{14} \mathrm{~W} \mathrm{~cm}^{-2}$. (b) The reconstructed FROG trace using the PCGP algorithm after 2000 iterations. (c) The spectra and spectral phases of the original (black solid line) and reconstructed signal pulses using Gaussian fitting (red dotted line) and PCGP algorithm (blue dashed line). (d) The electric field of the original (black solid line) and the reconstructed signal pulses using Gaussian fitting (red dotted line) and PCGP algorithm (blue dashed line).

simple reconstruction using Gaussian fitting fails to reproduce the original signal field when the signal spectrum covers a broad bandwidth.

The spectrum of the signal pulse reconstructed using Gaussian fitting is considerably narrower than that of the original signal pulse even if the single atom response is corrected. This is mainly due to the diffraction pattern appeared in the far-field spatial distributions. The FROG trace in figure 3(a) modulates up and down at the beginning of the pulse $(\tau<0)$, but it starts to diffract (near 0 fs) [16]. Thus, the simple approach that uses Gaussian fitting fails to retrieve the signal field.

In the real experiment, the accuracy of the reconstruction would be tested by comparing the reconstructed spectrum to the spectrum measured with a grating based spectrometer. However, the two spectra should be carefully compared. Since the petahertz optical oscilloscope is a time-domain measurement, the reconstructed spectrum will only contain the information of the pulse for a finite time span. On the contrary, the spectrum measured with the grating based spectrometer shows the spectrum of the entire signal pulse. This difference should be taken into account for the comparison.

\section{Conclusion}

In summary, we demonstrate the reconstruction of timedependent optical pulses using the phase retrieval approach in the petahertz optical oscilloscope. The petahertz optical oscilloscope measures an arbitrary optical field with all optical experiment setup which is more efficient than the 
attosecond streak camera. However, the technique should be carefully implemented for a broadband signal. With the phase retrieval approach that we present here, the broadband signal could be reconstructed very accurately.

\section{Acknowledgments}

This work was supported by IBS (Institute for Basic Science) under IBS-R012-D1.

\section{References}

[1] Itatani J, Quéré F, Yudin G L, Ivanov M Y, Krausz F and Corkum P B 2002 Attosecond streak camera Phys. Rev. Lett. 88173903

[2] Goulielmakis E et al 2004 Direct measurement of light waves Science 305 1267-9

[3] Kim K T, Zhang C, Shiner A D, Schmidt B E, Légaré F, Villeneuve D M and Corkum P B 2013 Petahertz optical oscilloscope Nat. Photonics 7 958-62

[4] Corkum P B 1993 Plasma perspective on strong field multiphoton ionization Phys. Rev. Lett. 71 1994-7

[5] Kienberger R et al 2004 Atomic transient recorder Nature 427 817-21

[6] Mairesse Y and Quéré F 2005 Frequency-resolved optical gating for complete reconstruction of attosecond bursts Phys. Rev. A 7111401
[7] Kane D J 1999 Recent progress toward real-time measurement of ultrashort laser pulses IEEE J. Quantum Electron. 35 421-31

[8] Kane D J 2008 Principal components generalized projections: a review [Invited] J. Opt. Soc. Am. B 25 A120

[9] Kim K T, Zhang C, Shiner A D, Kirkwood S E, Frumker E, Gariepy G, Naumov A, Villeneuve D M and Corkum P B 2013 Manipulation of quantum paths for space-time characterization of attosecond pulses Nat. Phys. 9 159-63

[10] Dudovich N, Smirnova O, Levesque J, Mairesse Y, Ivanov M Y, Villeneuve D M and Corkum P B 2006 Measuring and controlling the birth of attosecond XUV pulses Nat. Phys. 2 781-6

[11] Shafir D, Soifer H, Bruner B D, Dagan M, Mairesse Y, Patchkovskii S, Ivanov M Y, Smirnova O and Dudovich N 2012 Resolving the time when an electron exits a tunnelling barrier Nature 485 343-6

[12] Lee D G, Park J J, Sung J H and Nam C H 2003 Wave-front phase measurements of high-order harmonic beams by use of point-diffraction interferometry Opt. Lett. 28480

[13] Kim K, Nam C H and Kim K T 2016 Quantum path analysis for arbitrary optical-waveform measurements Phys. Rev. A 9333818

[14] Yudin G L and Ivanov M Y 2001 Nonadiabatic tunnel ionization: looking inside a laser cycle Phys. Rev. A 64 13409

[15] Yakovlev V S, Ivanov M and Krausz F 2007 Enhanced phasematching for generation of soft $\mathrm{x}$-ray harmonics and attosecond pulses in atomic gases Opt. Express 1515351

[16] Bertrand J B, Wörner H J, Bandulet H-C, Bisson É, Spanner M, Kieffer J-C, Villeneuve D M and Corkum P B 2011 Ultrahigh-order wave mixing in noncollinear high harmonic generation Phys. Rev. Lett. 10623001 